\title{
Does heritability hide in epistasis between linked SNPs?
}

European Journal of Human Genetics (2011) 19, 123; doi:10.1038/ejhg.2010.161; published online 6 October 2010

Much recent discussion addresses the question of 'missing heritability' in genome-wide association studies (GWAS). The problem can be illustrated using the example of human height. Classical pedigree studies show a high heritability of human height, in the order of $80 \%$. This is part of our everyday experience: tall parents tend to have tall children. GWAS has identified more than 40 loci associated with height, but these variants together explain only a small part of phenotypic variation. ${ }^{1}$ A number of hypotheses have been advanced to identify the source of the missing heritability, including large effects of rare variants and effects of copy-number variation. ${ }^{2}$

A conceptual difference between pedigree studies and GWAS does not appear to have been considered: pedigree-based heritability measures the phenotypic effects of much larger chunks of chromosome than GWAS-based heritability. This distinction can be illustrated with a simple example that elides complexities arising from diploidy. Consider two SNPS (A/T and G/C) in linkage equilibrium that are located $0.1 \mathrm{~cm}$ apart. The SNPs could, for example, encode two amino acid substitutions within a single protein. From the perspective of pedigree-based measures of heritability, the four haplotypes (AG, AC, TG and TC) are inherited as four alleles at a single locus, but from the perspective of GWAS these are biallelic polymorphisms at distinct loci. Suppose that the combinations AG and TC add a little bit extra to height but AC and TG subtract a little bit. Then, neither SNP will be correlated with height in GWAS, but the haplotypes, which are correlated with height, will be reliably transmitted from parents to offspring and will contribute to estimates of pedigreebased heritability. Put another way, the genetic effect on phenotype appears as part of the additive genetic variance in pedigree studies but as an unmeasured gene $\times$ gene interaction in GWAS.

The major constraint on measuring interactions in GWAS has been the very large number of possible interactions. If there are $10^{6} \mathrm{SNPs}$ on an array, then there are $5 \times 10^{11}$ pairs of SNPs. However, the number of pairs is a much more manageable $10^{6}$ if analysis is restricted to neighboring SNPs.

\section{CONFLICT OF INTEREST}

The author declares no conflict of interest.

David Haig

Department of Organismic and Evolutionary Biology, Harvard University, Cambridge, MA, USA E-mail:dhaig@oeb.harvard.edu

\footnotetext{
1 Weedon MJ, Frayling TM: Reaching new heights: insights into the genetics of human stature. Trends Genet 2008; 24: 595-603.

2 Manolio TA, Collins FS, Cox NJ et al: Finding the missing heritability of complex diseases. Nature 2009; 461: 747-753.
}

\section{Does the HSD17B10 gene escape from $\mathrm{X}$-inactivation?}

European Journal of Human Genetics (2011) 19, 123-124; doi:10.1038/ejhg.2010.192; published online 17 November 2010

We read with great interest the recent report by Garcia-Villoria et al ${ }^{1}$ regarding the expression of the HSD17B10 gene from the inactive X chromosome that was published in the European Journal of Human Genetics (Advance online publication, 28 July 2010; doi:10.1038/ejhg.2010.118).

It had been reported previously that a cluster of six genes, including the HSD17B10 (formerly $H A D H 2$ ) gene in $\mathrm{Xpl} 1.2$, escapes $\mathrm{X}$-inactivation. ${ }^{2}$ Subsequently, Carrel and Willard, in a more detailed study, ${ }^{3}$ showed that the escape of the HSD17B10 gene from $\mathrm{X}$-inactivation is not complete. The expression of the HSD17B10 gene and the surrounding genes from the inactive $\mathrm{X}$ chromosome (Xi) is summarized in Figure 1 (adapted from Ref. Yang et al ${ }^{4}$ ).

Two female patients heterozygous for HSD10 deficiency were the subjects of this present study ${ }^{1}$ in which skin fibroblast cultures were examined to determine the inactivation ratio of the normal and mutated X chromosomes. It appears that these studies were performed on cultures originating from a single biopsy from each patient. Mosaicism due to lyonization results in relatively large patches of skin with the same inactivated $\mathrm{X}$ chromosome, commonly illustrated by the coloration of calico cats. Thus, an analysis of cells from a single biopsy is probably not adequate to determine the $\mathrm{X}$ inactivation ratio. Analysis of a blood sample might be more informative.

In addition, the standard deviation (SD) appears to be relatively large, that is, $>15 \%$ of the mean value in most cases. This limitation

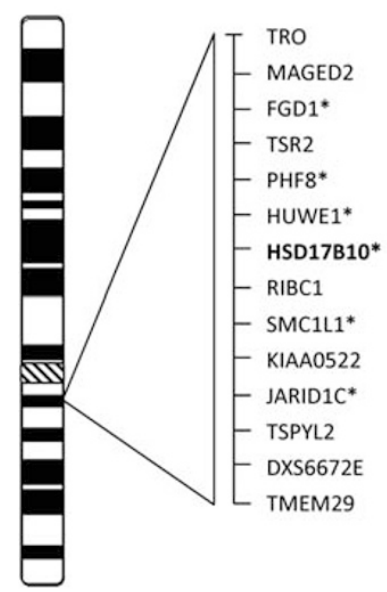

$\begin{array}{ccc}\mathrm{X}_{\mathrm{a}} & \mathrm{X}_{\mathrm{i}} & \mathrm{X}_{\mathrm{i}} \text { expression in hybrids tested } \\ + & - & 0 / 5 \\ + & - & 0 / 9 \\ + & + & 1 / 9 \\ + & + & 1 / 9 \\ + & - & 0 / 9 \\ + & + & 1 / 9 \\ + & + & 1 / 9 \\ + & + & 7 / 9 \\ + & + & 7 / 9 \\ + & + & 9 / 9 \\ + & + & 9 / 9 \\ + & - & 0 / 5 \\ + & - & 0 / 5 \\ + & - & 0 / 5\end{array}$

Figure 1 Expression of transcripts of the $H S D 17 B 10$ and surrounding genes from inactive $X(\mathrm{Xi})$ hybrids. Samples scored as positive are expressed at least $>10 \%$ of the $X a$ levels, and their number is shown as the numerator. The total number of hybrids tested is shown as the denominator. Genes with mutation(s) $)^{5}$ or copy number variation $(\mathrm{CNV})^{6}$ causing mental retardation are marked with asterisk. 